\title{
EPIPHYSEAL TRANSPLANT: HARVESTING TECHNIQUE OF THE PROXIMAL FIBULA BASED ON THE ANTERIOR TIBIAL ARTERY
}

\author{
MARCO INNOCENTI, M.D., ${ }^{*}$ LUCA DELCROIX, M.D., and GIANMARIA FEDERICO ROMANO, M.D.
}

\begin{abstract}
Epiphyseal transplants in children were introduced into clinical practice about 20 years ago. Among possible donor sites, the proximal fibula is definitely the most popular choice, and has been used mainly for reconstruction of the proximal humerus and distal radius. Provided that an adequate blood supply both to the physis and to the diaphysis must be restored in order to obtain acceptable axial growth of the transferred fibula and a bone fusion at the osteotomy site, the choice of feeding pedicle is still a controversial issue. Our contribution involves a homogeneous series of 24 patients under 11 years of age who had skeletal reconstruction in the upper limb by means of a vascularized transfer of the proximal fibula based on the anterior tibial artery. The aim of the present paper is to describe in detail the harvesting technique which has been partially modified and gradually refined in our 10-year experience. (c) 2005 Wiley-Liss, Inc. Microsurgery 25:284-292, 2005.
\end{abstract}

The possibility of transferring a bone autograft while preserving growth potential has been investigated for many years by the international scientific community. This procedure is of great value in dealing with epiphyseal bone reconstruction in skeletally immature patients, where the prevention of future limb-length discrepancy is a very relevant issue.

Experimental experience pointed out the importance of a blood supply to the growth plate. Thus, a vascularized graft is mandatory in order to achieve the desired results in terms of growth and physis survival. ${ }^{1-7}$ The next step was the selection of an appropriate donor site and the extensive study of its vascular supply. Some authors ${ }^{8}$ suggested the use of the iliac crest and the distal part of the scapula. These bone segments, however, are not true epiphyses, and they are anatomically identified as apophyses. Therefore, their transfer to an intra-articular location provides unpredictable results due to the absence of a real articular surface. Furthermore, both the scapula and iliac crest are flat bones and cannot be used when a portion of the diaphysis has to be reconstructed.

In our opinion, the proximal fibula is a better option. This bone provides an epiphysis and a variable amount of the adjoining diaphysis. In addition, the fibula, due to its shape and dimensions, is the ideal choice in cases of skeletal reconstruction in the upper limb.

Over the period from $1992-2004,{ }^{9-11} 24$ vascularized proximal fibula epiphyseal transplants in children were performed using the anterior tibial artery as vascular pedicle (Fig. 1A, B). The aim of the present paper is to describe in detail the harvesting technique in cases

Reconstructive Microsurgery Unit, Department of Orthopedics, Azienda Ospedaliera Careggi, Centro Traumatologico Ortopedico, Florence, Italy

${ }^{*}$ Correspondence to: Marco Innocenti, M.D., Reconstructive Microsurgery Unit, Azienda Ospedaliera Careggi, Centro Traumatologico Ortopedico, Largo Palagi 1, 50139 Florence, Italy. E-mail: m.innocenti@agora.it

Received 28 February 2005; Accepted 8 March 2005

Published online 2 June 2005 in Wiley InterScience (www.interscience. wiley.com). DOI: 10.1002/micr.20130 of transfer of the fibula, incorporating the proximal epiphysis and preserving an adequate blood supply both to the growth plate and to the diaphysis.

\section{SURGICAL TECHNIQUE}

The patient is placed supine, with the donor extremity flexed at the hip and the knee. A pneumatic tourniquet is used to maintain a bloodless field. A straight longitudinal incision is centered over the space between the tibialis anterior muscle (TA) and the extensor digitorum longus muscle (EDL) and is prolonged proximally, following the tendon of the biceps femoris muscle (Fig. 2A, B).

The superficial fascia is opened, and the dissection is carried out in the intermuscular plane between TA and EDL, to expose the tibialis anterior neurovascular bundle (Fig. 3). The common peroneal nerve is then identified proximally at the level of the fibular neck and protected.

The dissection of the vascular pedicle must preserve the peroneal nerve as well as the delicate network of musculo-periosteal branches, which are crucial in order to supply the fibular diaphysis. Thus, it is recommended to start the exposure of the neurovascular bundle fairly distally, since in the lower third of the leg, either the motor branches of the deep peroneal nerve or the feeding vessels to the fibular diaphysis are sporadic, and it is easier for the surgeon to identify the correct plane of dissection (Fig. 4).

The origin of the extensor digitorum longus (EDL), peroneus longus $(\mathrm{PL})$, and brevis $(\mathrm{PBr})$ muscles are incised sharply and retracted laterally and downward, in order to expand the surgical field (Fig. 5A, B). Such an incision should be done at about $2 \mathrm{~cm}$ distal to the fibular tip, and a muscular cuff is therefore left intact surrounding the fibular epiphysis, in order to reduce the risk of damaging the recurrent epiphyseal branch which supplies the growth plate. 


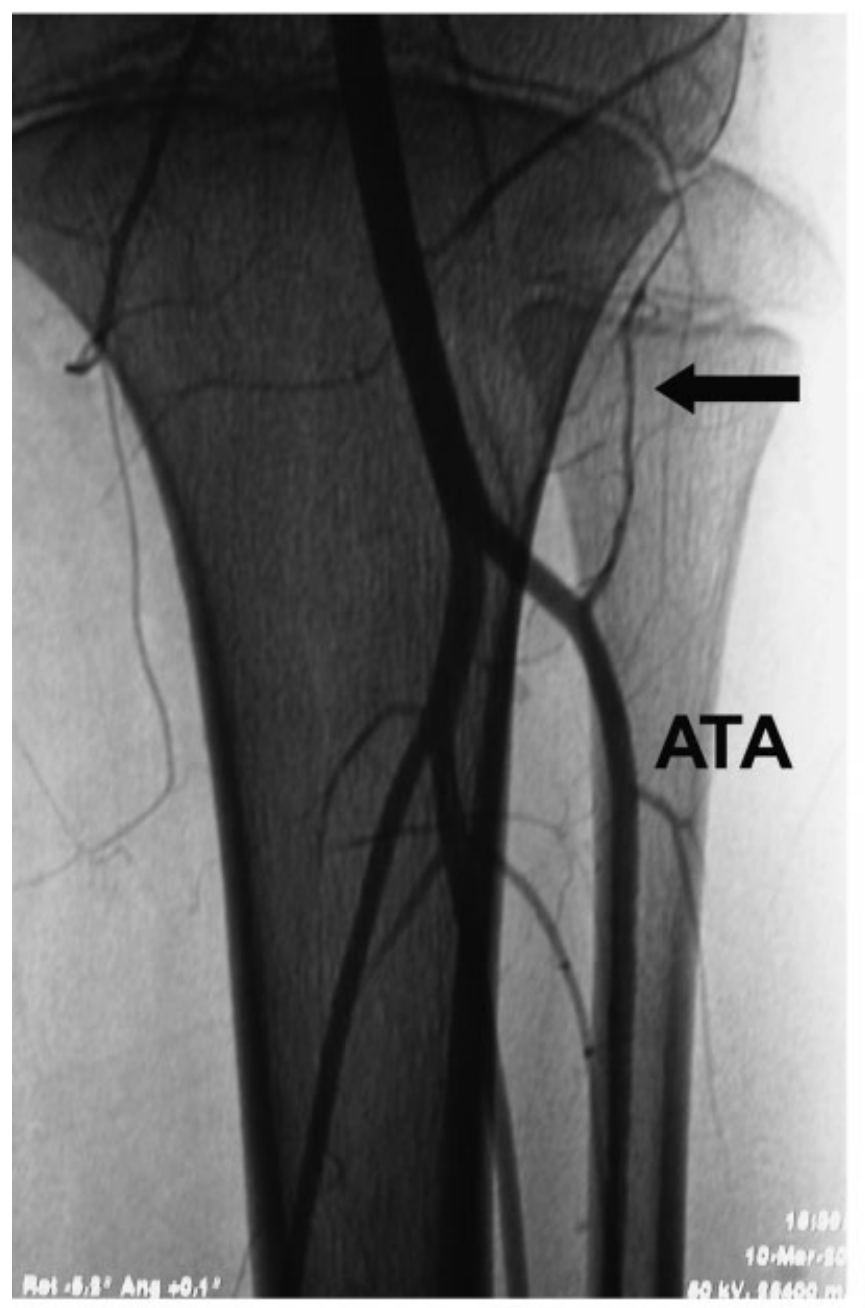

A

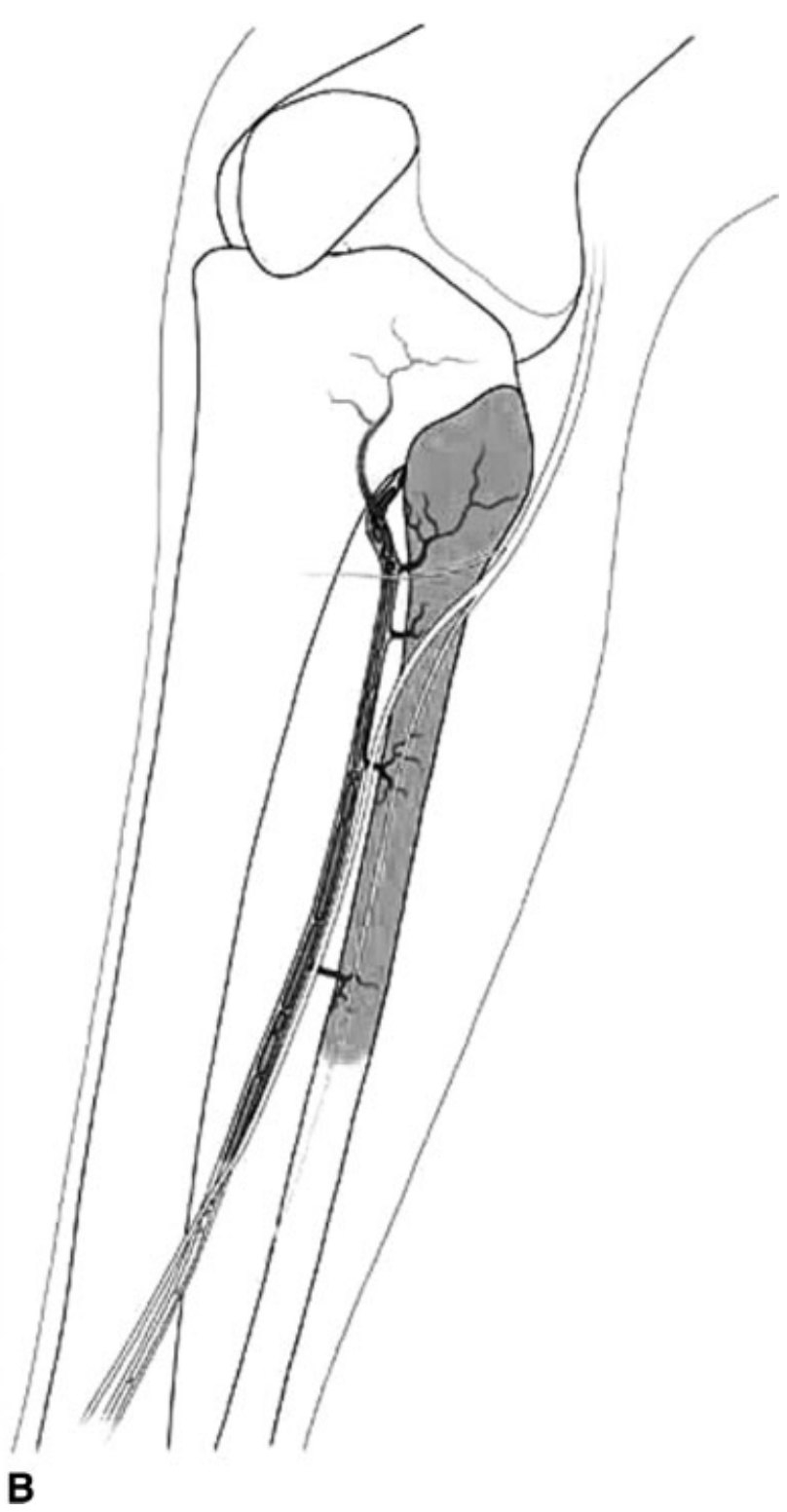

Figure 1. A. Preoperative angiogram: anterior tibial artery supplies proximal growth plate of the fibula by means of a recurrent epiphyseal branch (arrow) B. Anterior tibial artery provides also the periosteal blood supply to proximal two thirds of diaphysis by means of tiny musculo periosteal branches.

The deep peroneal nerve follows the anterior tibial artery in the intermuscular space, and sends many motor branches to the muscles of the anterior compartment of the leg. The intricate network of motor branches surrounds the vessels in a variable fashion, and the dissection must be meticulous (Fig. 6). In some instances, however, it is impossible to save all the muscular branches, and some of them must be intentionally severed by the surgeon in order to free up the vascular pedicle. In this case, a neurorraphy or direct neurotization should be performed in order to reduce the risk of muscular impairment. The dissection proceeds from distal to proximal, completing the dissociation of the nerve from the vascular bundle.

Tiny periosteal vessels lie on the anterior portion of the interosseous membrane and reach the fibular shaft perforating the EDL and PL muscles, and therefore must be carefully preserved. This goal is better achieved if the interosseous membrane is sharply detached from its tibial insertion, so that the vascular pedicle and its branches lie undisturbed in their natural bed (Fig. 7A, B). The periosteal branches are so small that they cannot 


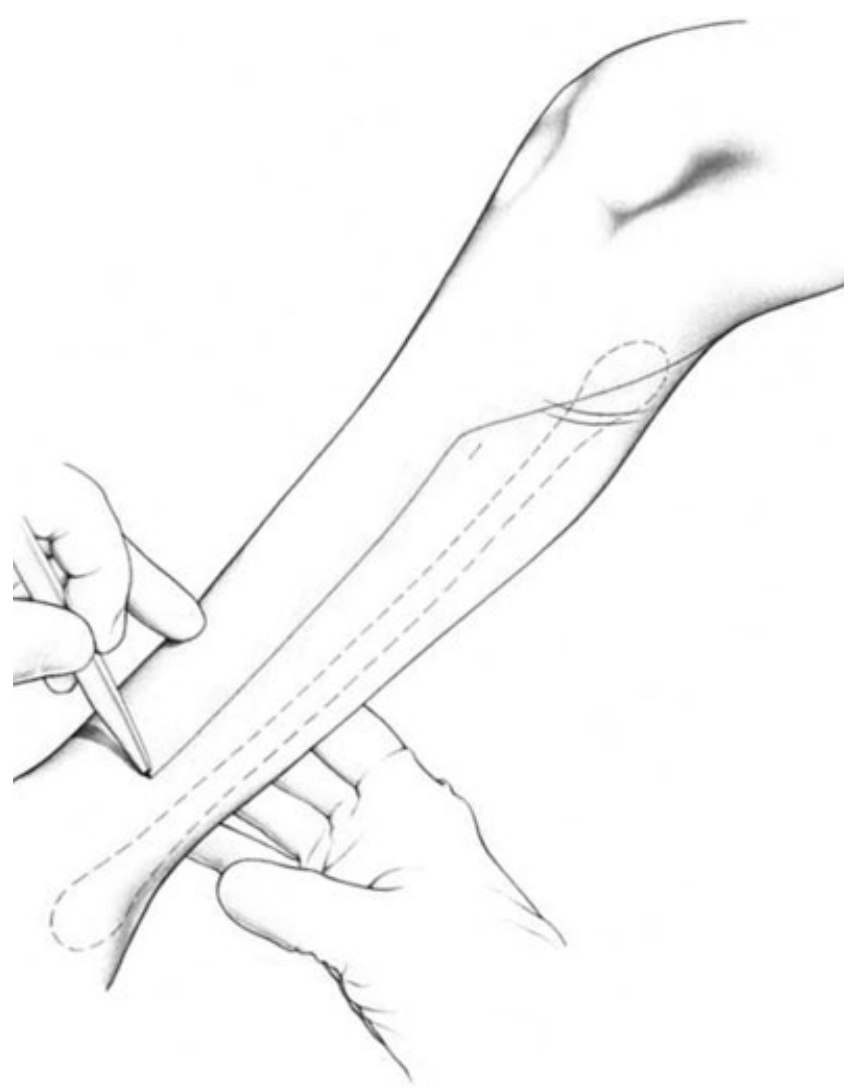

A

Figure 2. Artistic drawing (A) and clinical picture (B) illustrating the anterolateral approach to fibula.

be safely dissected from the muscular fibers: a longitudinal strip of muscle should therefore be left attached to the interosseous membrane.

At this stage of the procedure, the fibula is almost completely exposed by an anterolateral approach, and the distal osteotomy of the fibula can be performed at the desired level (Fig. 8A, B). The periostium is usually abundant in children, and it is highly recommended to harvest an extra portion of it which should overlap the osteotomy site, once the fibula has been transferred to the recipient bone (Fig. 9).

The proximal tibiofibular joint is then opened, and the lateral collateral ligament is severed as distally as possible. The tendon of the biceps femoris muscle is longitudinally divided: the posterior strip is harvested with the fibula, and it can be used to stabilize the epiphysis in the recipient joint (Fig. 10A, B), while the anterior portion of the tendon is used to reinforce the lateral collateral ligament to provide sufficient stability to the knee joint. The descending genicular artery must be ligated, and the soft-tissue release is then completed.

Moving again to the interosseous space, the final part of the dissection is performed. The recurrent

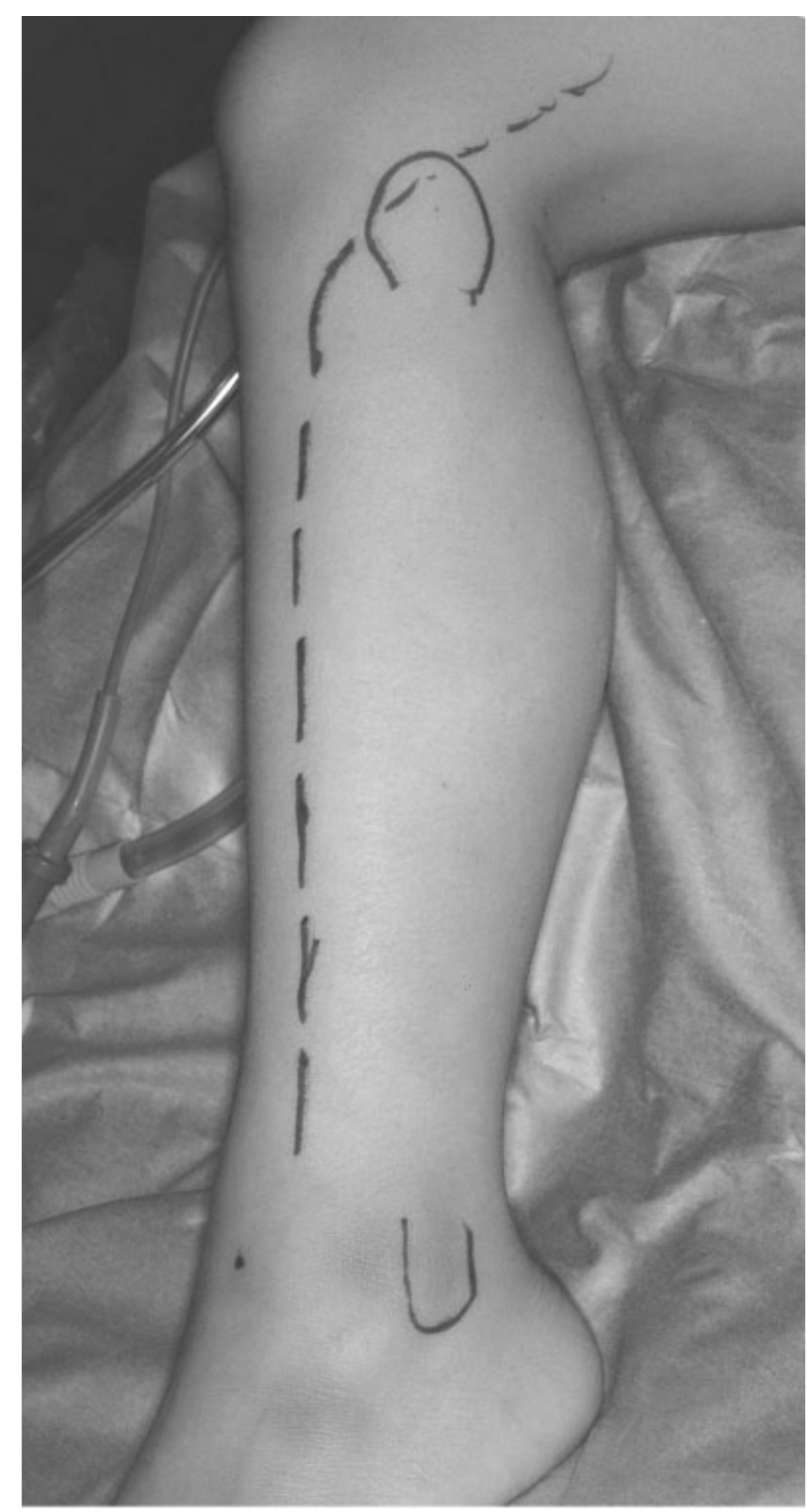

B

epiphyseal branch of the anterior tibial artery rises about $1 \mathrm{~cm}$ distal to its origin and perforates the muscular cuff surrounding the epiphysis. Such a muscular cuff should therefore be left intact, in order to prevent damage to the tiny epiphyseal artery. The collateral medial branches are then legated and the fibular head is subluxated laterally, allowing exposure of the origin of the anterior tibial artery in the posterior compartment. The distal part of the pedicle is divided, and the tourniquet is released. Bleeding should be observed either from the muscular cuff surrounding the physis or from the sectioned diaphysis, denoting sufficient blood supply (Fig. 11A). 


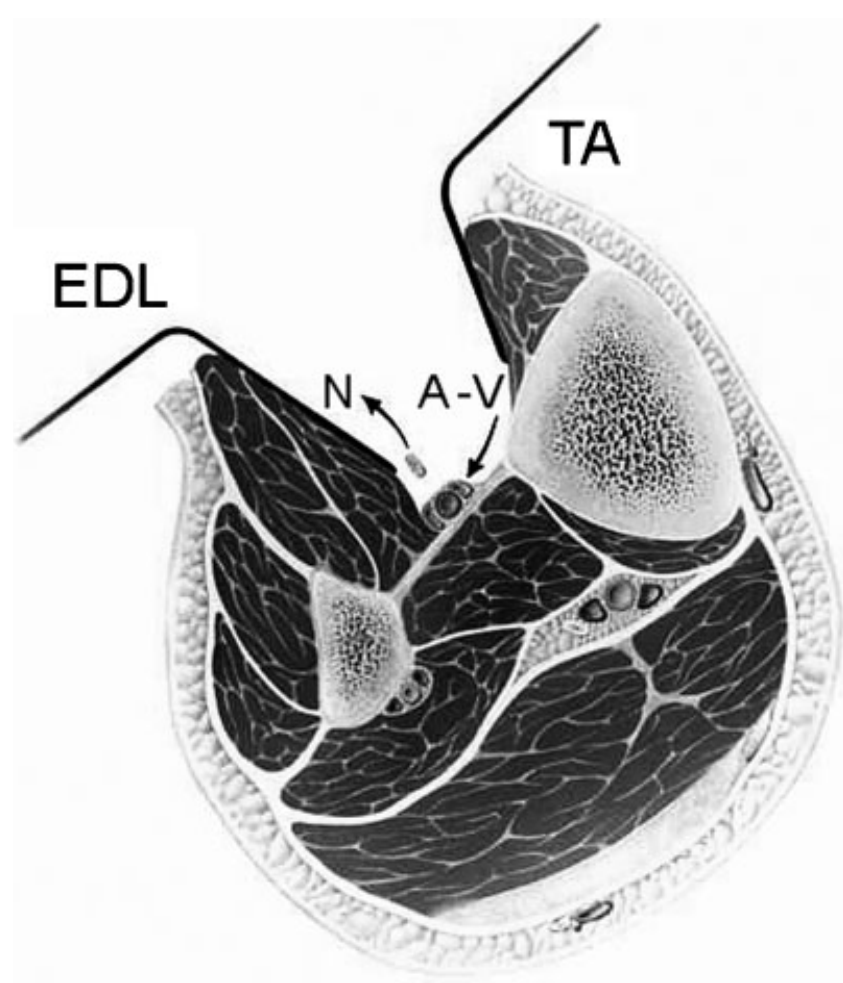

Figure 3. Dissection in space between tibialis anterior (TA) and extensor digitorum longus (EDL) muscles allows for direct approach to tibialis anterior neurovascular bundle. $N=$ nerve, $A=$ artery, $V=$ vein.

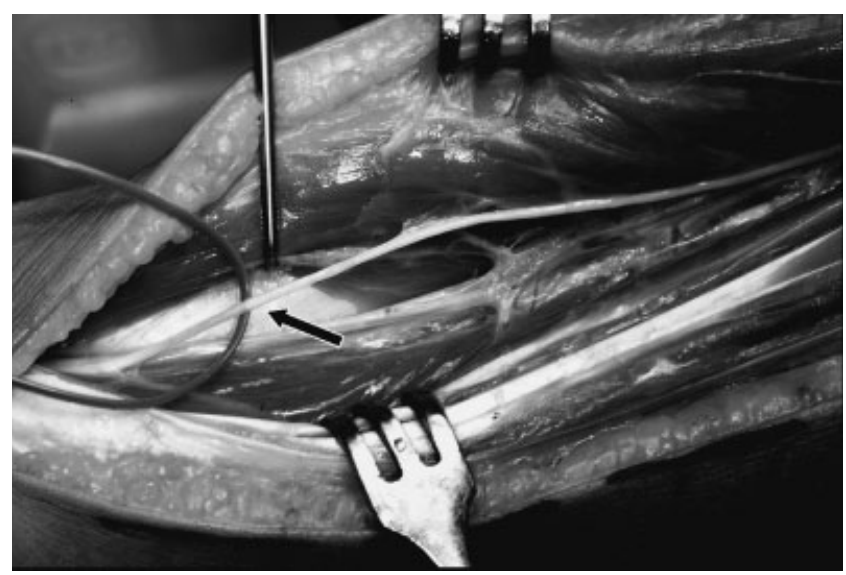

Figure 4. Dissection of peroneal nerve (arrow) from anterior vascular network is easier in distal portion of operating field, since at this level, motor branches are sporadic. For this reason, it is advisable to start dissection fairly distally.

After adequate reperfusion of the growth plate, the anterior tibial artery and veins can be legated at their origin, and the bone can be harvested (Fig. 11B).

After harvest of the fibula, the lateral stability of the knee joint must be restored. We achieved excellent results reinforcing the lateral collateral ligament with the

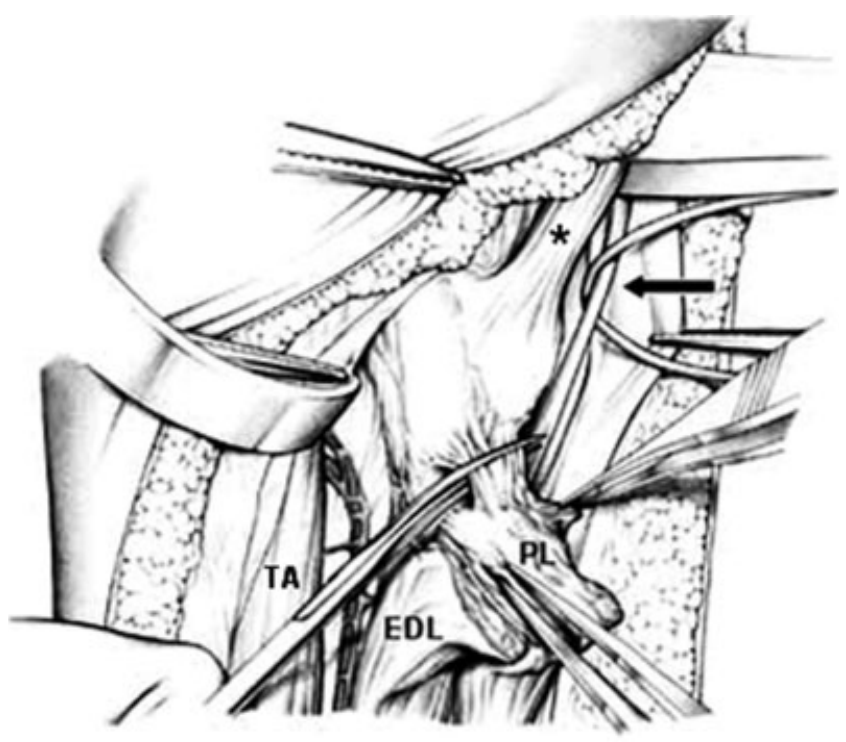

A

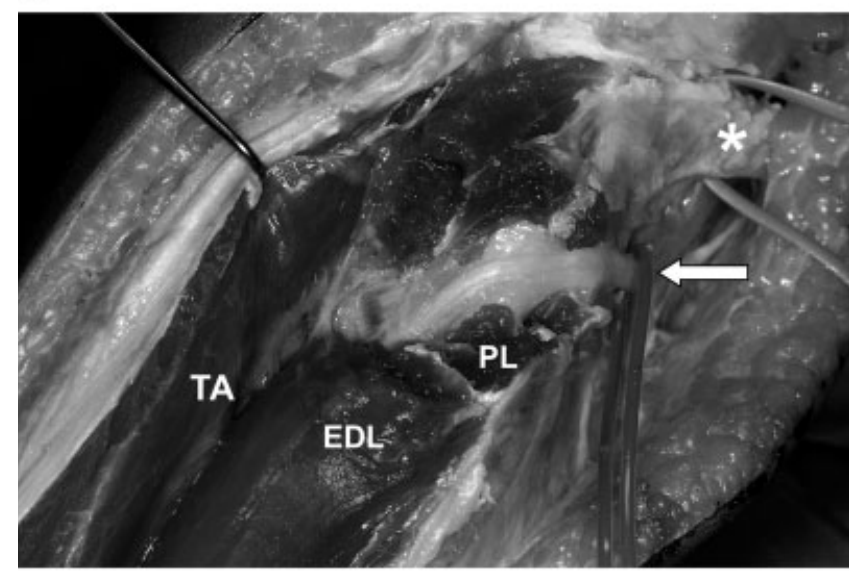

B

Figure 5. Artistic drawing (A) and clinical picture (B) illustrating details of the proximal dissection: peroneus longus (PL) and extensor digitorum longus muscles (EDL) are sharply detached from fibula at level of the peroneal nerve (arrow). Proximal muscular cuff must be preserved since it contains recurrent epiphyseal branch of anterior tibial artery. *insertion of biceps femoris tendon.

residual strip of the biceps femoris tendon, fixing it to the lateral portion of the tibia by means of transosseous stitches. All our patients recovered adequate joint stability, and are able to use the donor limb without limitations.

\section{DISCUSSION}

The introduction of this procedure into clinical practice goes back to the early 1980 s, when a few surgeons replaced either the distal radius or the proximal humerus in children using an autologous fibula incorporating the proximal epiphysis. The few reports in the 


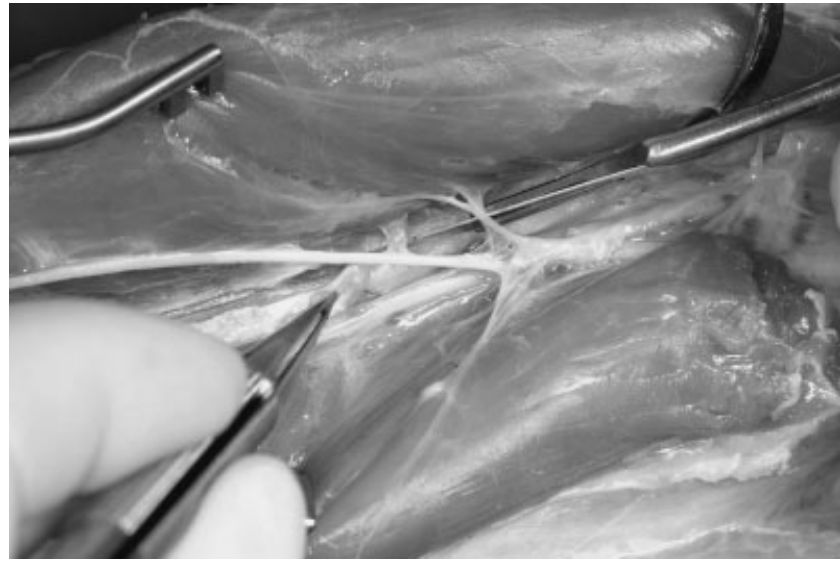

Figure 6. During proximal dissection, many motor branches of peroneal nerve cross operating field, and they must be carefully preserved.

literature provide varying results, in terms of growth or physis survival. The choice of the pedicle is a crucial point and has been a controversial issue. Some authors ${ }^{12}$ harvested the fibula supplied by the peroneal vessels, believing that such a pedicle would adequately vascularize the growth plate as well as the shaft of the bone. This opinion is in contrast with classical anatomical studies, ${ }^{13}$ which demonstrate that until the end of growth, the diaphyseal and the epiphyseal vascular networks are independent and not connected to each other. Further anatomical investigations on the proximal fibula vascular supply ${ }^{14}$ demonstrated that the epiphysis is vascularized by the descending genicular artery and a recurrent branch from the anterior tibial artery.

For this reason, some authors assembled the peroneal artery and one epiphyseal vessel in a variable fashion, ${ }^{8,15,16}$ with the aim of guaranteeing optimal perfusion both to the shaft and epiphysis. Such a surgical solution, however, is technically demanding and increases operative time. Furthermore, the necessity of multiple anastomosis increases the risk of microvascular complications.

In 1988, Taylor et al. described an anterolateral approach to the fibula and suggested the possibility of harvesting the proximal two thirds of this bone, based on the anterior tibial artery alone. ${ }^{17}$ This option was supported by anatomical investigations, which revealed a significant contribution of the anterior tibial artery to the periosteal diaphyseal network of the fibula by means of tiny branches which rise from the proximal and middle portions of this artery. The constant presence of the recurrent epiphyseal branch makes the anterior tibial artery the ideal pedicle to provide adequate blood supply to both the diaphysis and epiphysis of a growing

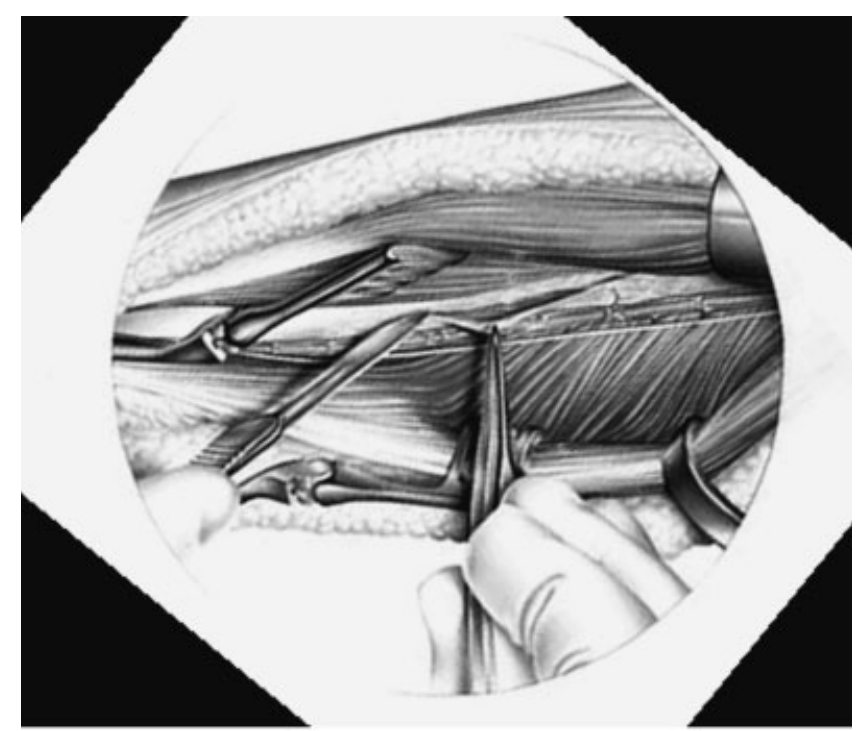

A

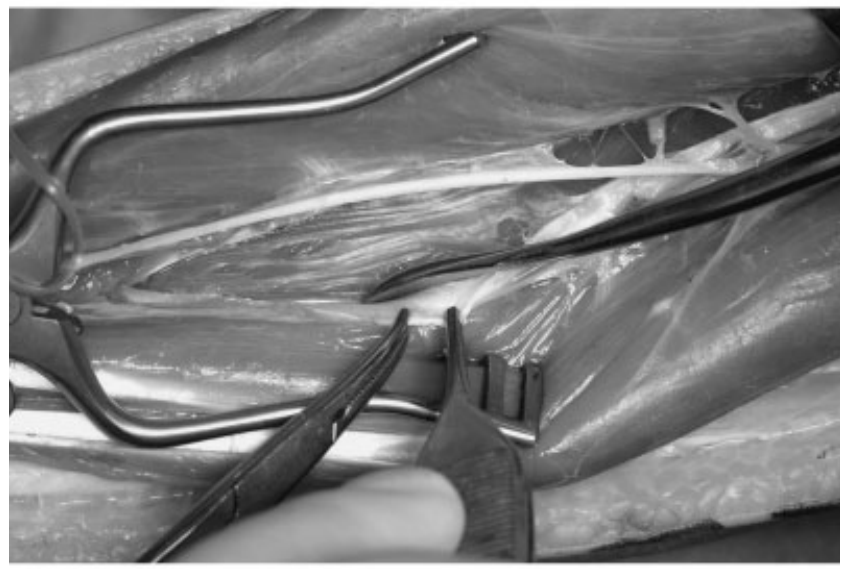

B

Figure 7. In order to protect vascular pedicle, it is suggested to detach interosseous membrane from tibial insertion (A), and preserve a muscular cuff on interosseous membrane, which contains small musculo-periosteal branches to diaphysis (B).

fibula. The major technical problem related to the choice of anterior tibial artery as the vascular pedicle of such a graft is the shortness of the proximal stump, which should be augmented with a vein graft. In order to reduce the ischemia time of the growth plate and to minimize risks related to the vein graft, we modified the original technique, adopting a reverse flow model which in our experience significantly simplifies the procedure. As reported in the literature ${ }^{18,19}$ and confirmed by clinical experience, the venous reflow can be inverted, provided that the small shunts which bypass the valves are preserved during the dissection of the vascular bundle. Usually, only one of the two comitantes veins provides adequate reflow, and the surgeon should be aware of which vein is the better choice for the anasto- 


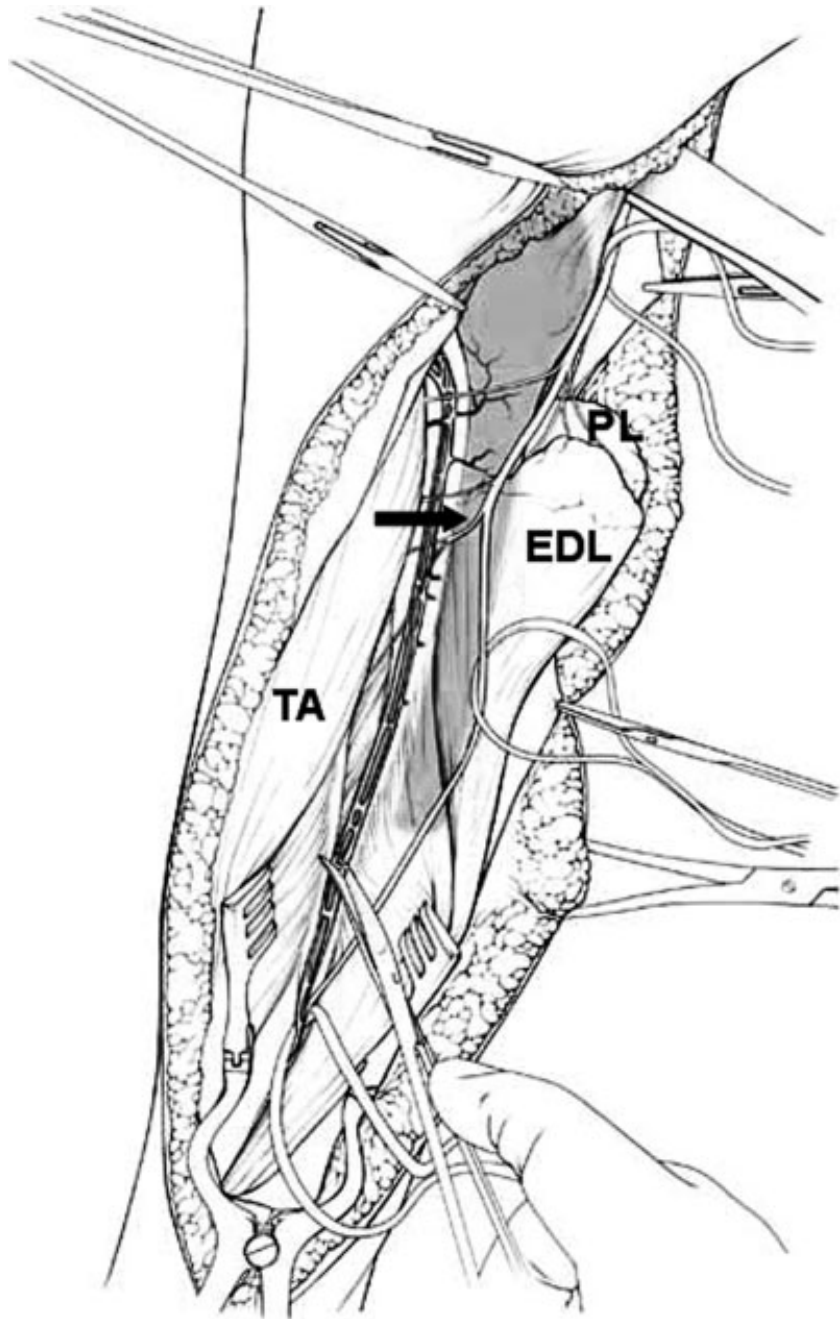

A

Figure 8. A: Fibula is almost completely exposed by anterolateral approach (TA, tibialis anterior m.; PL, proneus longus m.; EDL, extensor digitorum longus $m$.). B: Proximal motor branches of peroneal nerve are isolated and preserved (arrow).

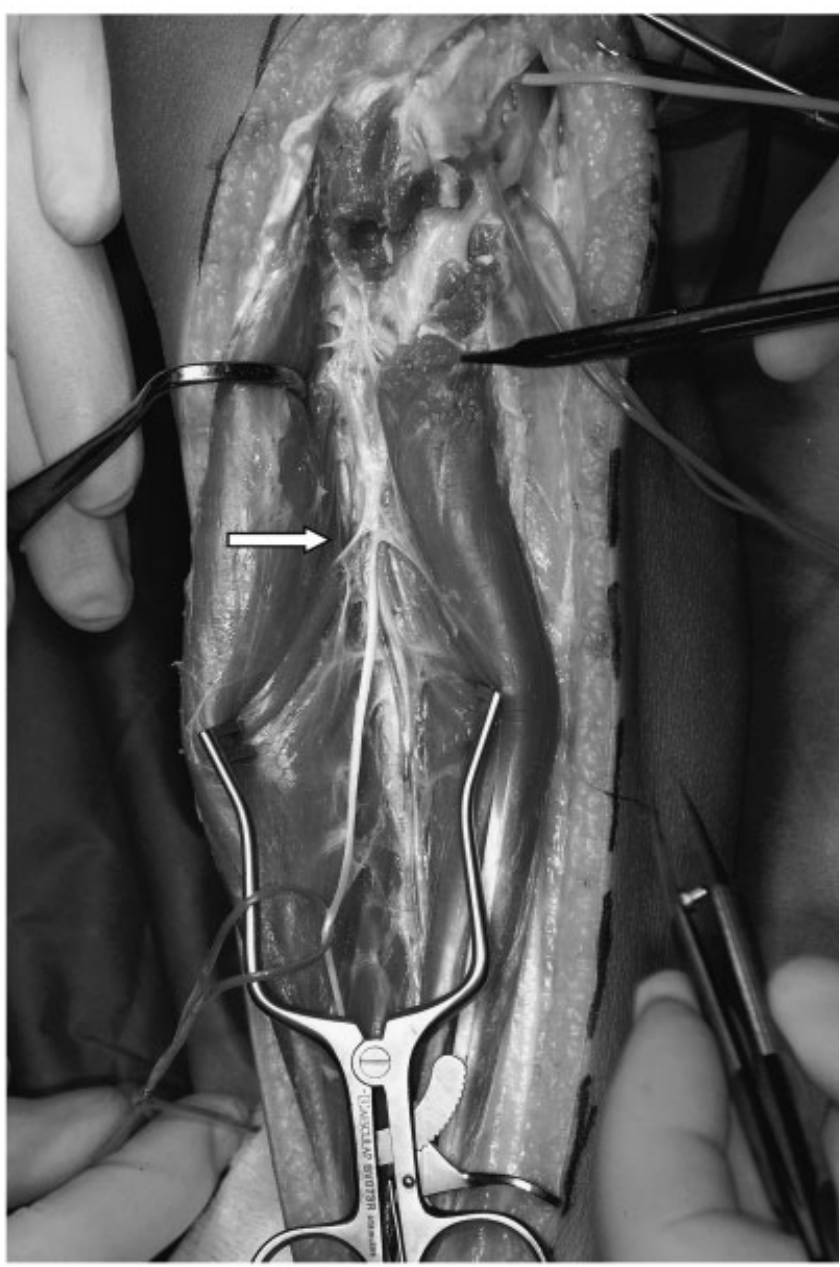

B mosis in the recipient site. The reverse-flow model provides a long distal pedicle which can be easily anastomosed to the recipient vessels.

Although the harvest of the fibula supplied by the anterior tibial artery is technically demanding and should be reserved only for skilled microsurgeons, the described procedure is a very useful tool in skeletal reconstruction in growing patients. In our opinion, this technique, if meticulously performed, is sufficiently reliable to be introduced into clinical practice. In our series, all grafts survived and united with the recipient bone within 3 months of surgery, denoting enough blood supply to the diaphysis. The physis proved viable in $85 \%$ of controlled patients, with an average growth rate $/$ year equal to $0.92 \mathrm{~cm}$. (Fig. 12A, B).

The choice of anterior tibial artery as single pedicle for such a graft significantly reduces the ischemia time of

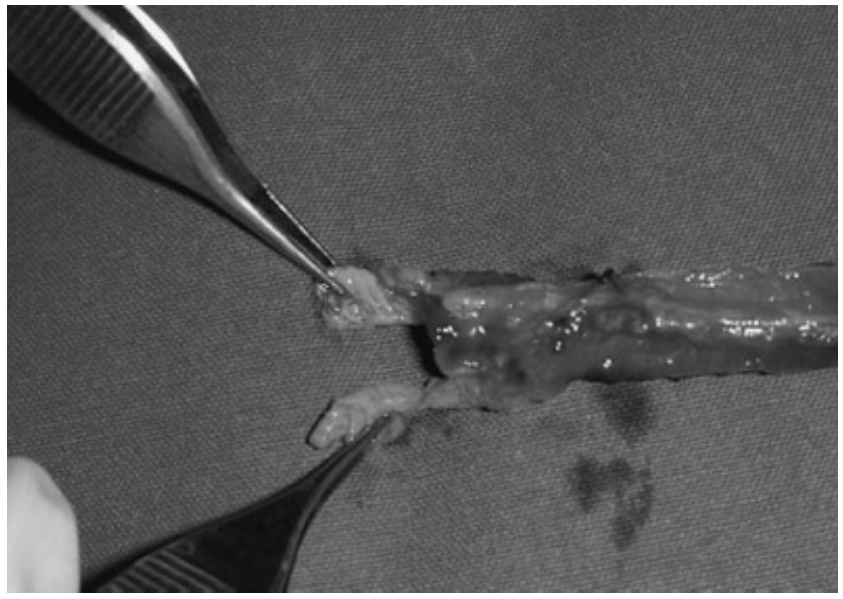

Figure 9. A portion of periosteoum should overlap osteotomy site in order to facilitate bone fusion. 


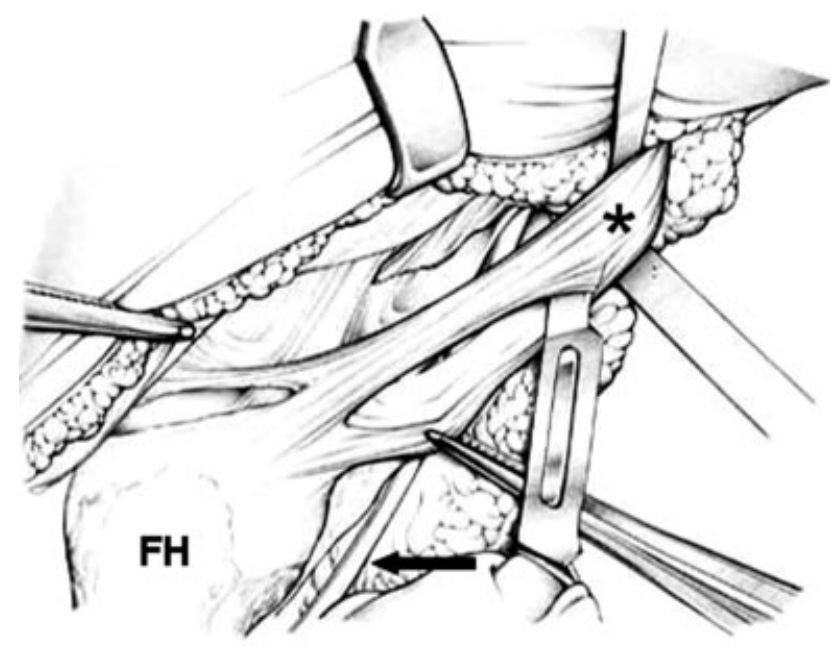

Figure 10. Tendon of biceps femoris muscle (asterisk) is longitudinally divided from fibular head (FH), and posterior strip is harvested with fibula. Posterior tendon strip can be used to stabilize epiphysis in recipient joint, and anterior strip is used to reinforce lateral collateral ligament, achieving adequate knee stability. Peroneal nerve (arrow).

the bone during the operation, and thus prevents possible ischemic damage to the growth plate. In addition, the reverse-flow design of the flap provides a very long distal pedicle, and makes a vein graft unnecessary.

The only complication that has been observed in the donor site pertains to the function of the deep peroneal nerve. As already mentioned, the dissociation of the nerve from the vascular bundle is a very difficult step of the harvesting procedure, and some muscle impairment should be expected postoperatively. In our series, about two thirds of the patients presented a transient palsy of the deep peroneal nerve, which recovered during the following month except for three patients, who suffered permanent palsy of the extensor digitorum longus and extensor hallucis longus muscles. By contrast, we never observed knee-joint instability after the described procedure of reconstruction of the lateral collateral ligament.

\section{CONCLUSIONS}

In our opinion, the anterior tibial artery is the firstchoice pedicle in cases of vascularized transfer of the proximal fibula in skeletally immature patients. This option provides a single pedicled flap which proved to be adequately vascularized in either the diaphyseal or epiphyseal portion. The described procedure proved effective in the reconstruction of the bone defect and in the prevention of future limb-length discrepancy, providing continuous and significant axial growth of the transferred fibula.

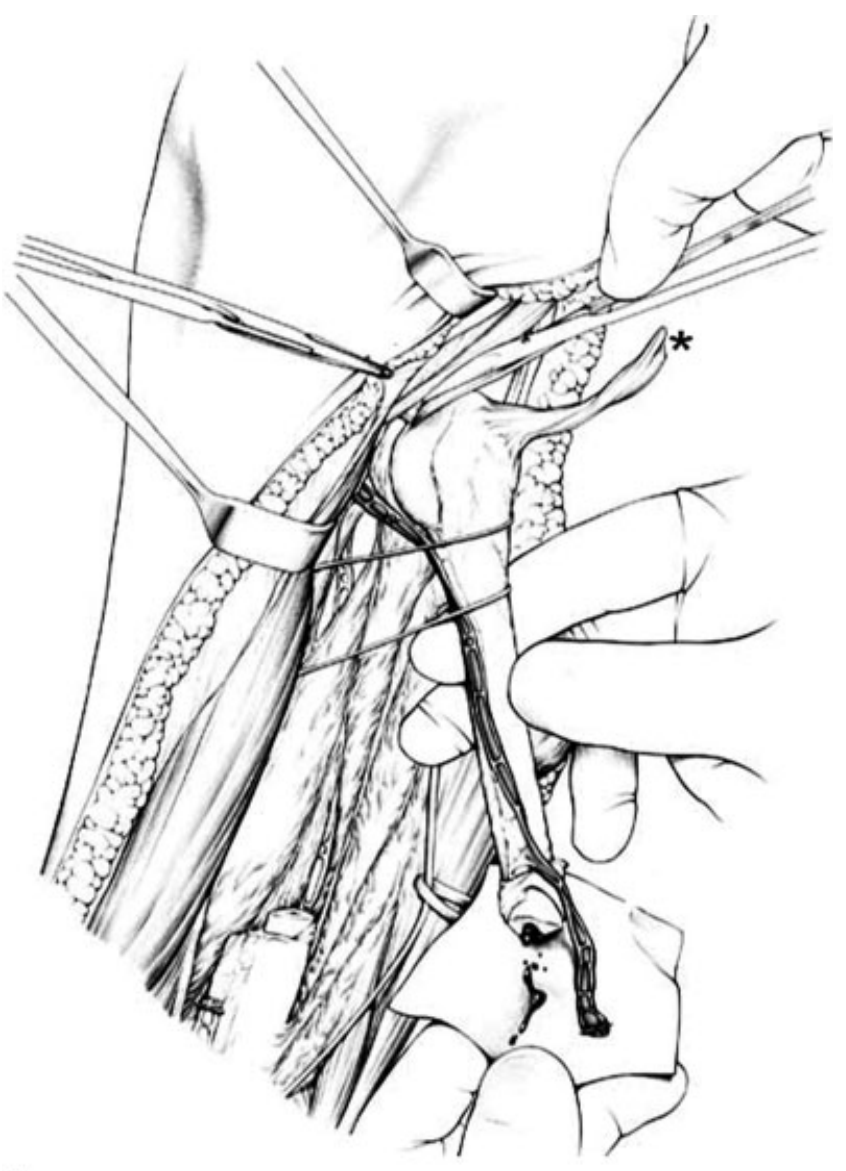

A

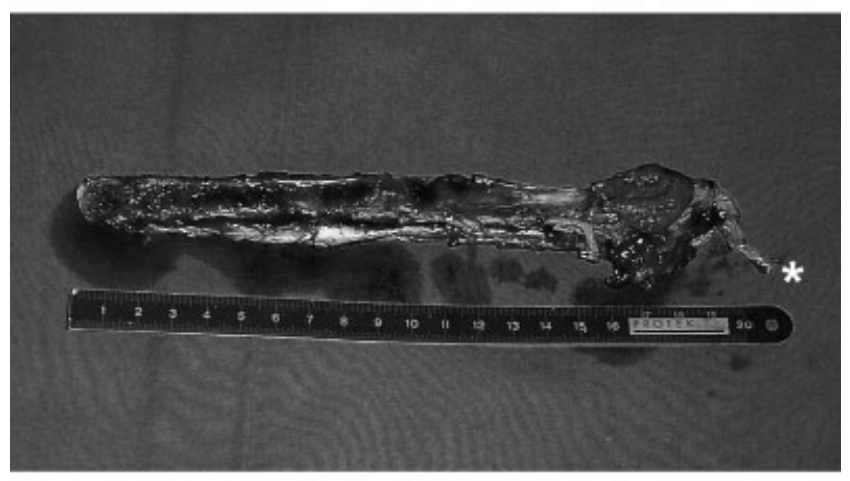

B

Figure 11. After distal resection of shaft, fibular head is dislocated laterally to expose origin of anterior tibial artery. A: At release of tourniquet, bleeding should be observed both from distal osteotomy and muscular cuff surrounding proximal physis. B: Graft includes proximal epiphysis, a strip of biceps femoris tendon, and approximately 2/3 of diaphysis. Asterisk = biceps femoris tendon.

Although some technical steps could be further improved, the harvesting technique has been sufficiently tested and standardized to be suggested for clinical practice. 


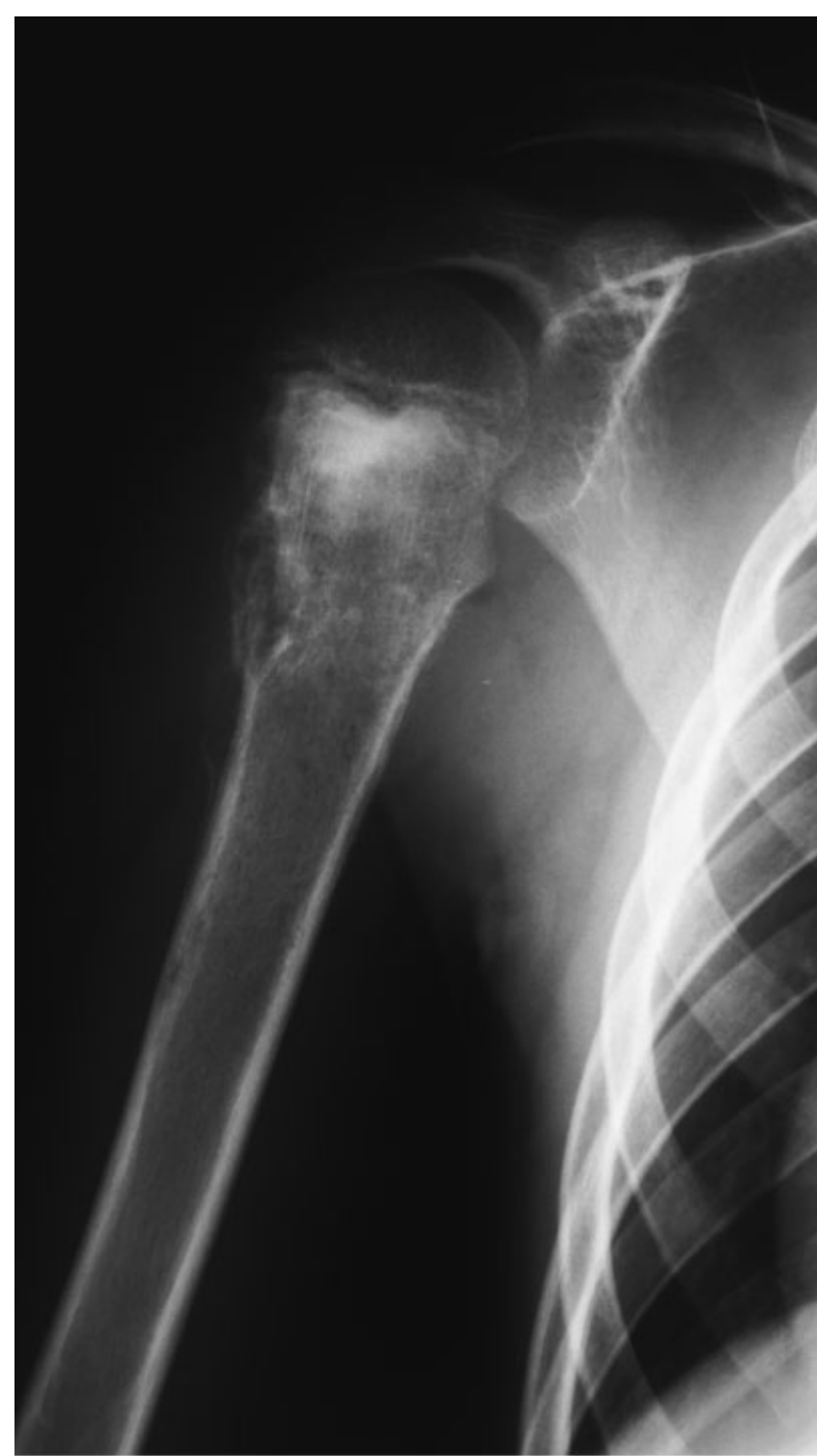

A

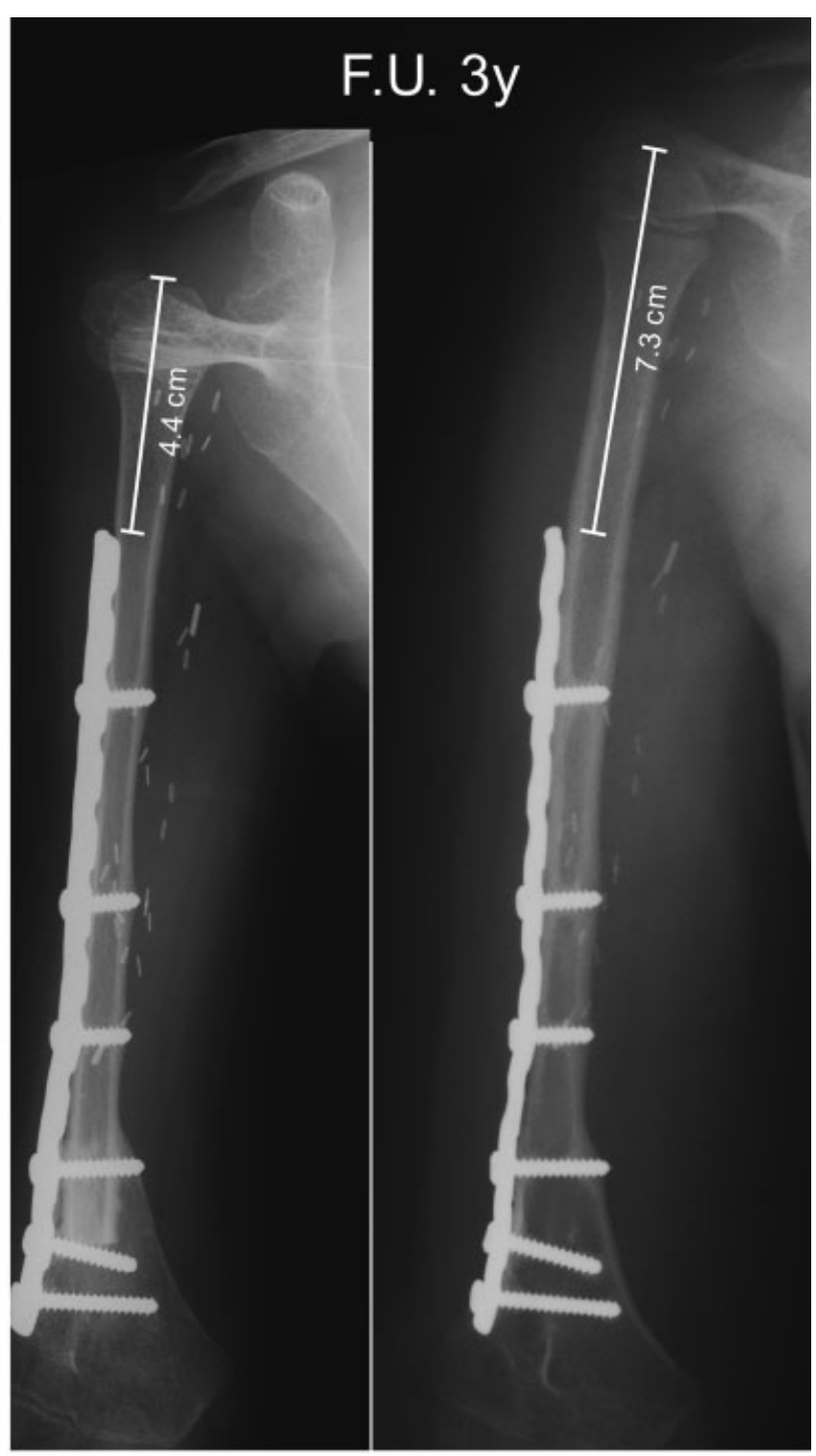

B

Figure 12. A: Osteosarcoma of humerus in 8-year-old male. After resection of proximal 2/3 of humerus (17 cm), one-stage reconstruction was achieved with vascularized proximal fibula epiphyseal transplant. B: Three years after surgery, longitudinal growth was $2.9 \mathrm{~cm}$.

\section{REFERENCES}

1. Bowen CVA. Experimental free vascularized epiphyseal transplants. Orthopedics 1986;9:893-898.

2. Bowen CVA, Ethridge CP, O'Brien BM, Frykman GK, Gumley GJ. Experimental microvascular growth plate transfers. Part 1-investigation of vascularity. J Bone Joint Surg [Br] 1988;70: 305-310.

3. Bowen CVA, O'Brien BM, Gumley GJ. Experimental microvascular growth plate transfers. Part 2-investigation of feasibility. $\mathbf{J}$ Bone Joint Surg [Br] 1988;70:311-314.

4. Nettelblad H, Randolph MA, Weiland AJ. Physiologic isolation of the canine proximal fibular epiphysis on a vascular pedicle. Microsurgery 1984;5:98-101.

5. Nettelblad H, Randolph MA, Weiland AJ. Free microvascular epiphyseal plate transplantation. An experimental study in dogs. J Bone Joint Surg [Am] 1984;66:1421-1430.
6. Donski PK, Carwell GR, Sharzer LA. Growth in revascularized bone grafts in young puppies. Plast Reconstr Surg 1979;64: 239-243.

7. Donski PK, O'Brien BM. Free microvascular epiphiseal transplantation: an experimental study in dogs. Br J Plast Surg 1980;33:169-178.

8. Wood MB, Gilbert A. Microvascular bone reconstruction. London: Martin Dunitz; 1997. 85 p.

9. Innocenti M, Ceruso M, Lauri G, Angeloni R, Manfrini M, Capanna R, Camnasio F. Free vascularized growth plate transplantation in a growing child. In: Limb salvage current trends. Proceedings. 7th International Symposium on Limb Salvage. 1993. p $423-430$

10. Innocenti $M$, Ceruso $M$, Manfrini $M$, Angeloni R, Lauri G, Capanna R, Bufalini C. Free vascularized growth plate transfer after bone tumor resection in children. J Reconstr Microsurg 1998;14:137-143. 
11. Innocenti M, Ceruso M, Delcroix L. Vascularized epiphyseal transfer in upper limb skeletal reconstruction in children. Indications and operative technique. In: Schuind F, editor. Advances in upper and lower extremity microvascular reconstructions. Singapore: World Scientific; 2002. p 90-105.

12. Pho RW, Patterson MH, Kour AK, Kumar VR. Free vascularized epiphyseal transplantation in upper extremity reconstruction. J Hand Surg [Am] 1988;13:440-447.

13. Trueta J. The role of the vessels in osteogenesis. J Bone Joint Surg [Br] 1963;45:402-404.

14. Bonnel F, Lesire M, Gomis R, Allieu Y, Rabischong P. Arterial vascularization of the fibula microsurgical transplant techniques. Anat Clin 1981;3:13-23.
15. Zhong-Wei C, Guang-Jian Z. Epiphyseal transplantation. In: Pho RW, editor. Microsurgical technique in orthopaedics. London: Butterworths; 1988. p 121-128.

16. Tsai TM, Ludwig L, Tonkin M. Vascularized fibular epiphyseal transfer: a clinical study. Clin Orthop 1986;210: 228-234.

17. Taylor GI, Wilson KR, Rees MD, Corlett RJ, Cole WG. The anterior tibial vessels and their role in epiphyseal and diaphyseal transfer of the fibula: experimental study and clinical applications. Br J Plast Surg 1988;41:451-469.

18. Lin SD, Lai CS, Chiu CC. Venous drainage in the reverse forearm flap. Plast Reconstr Surg 1984;74:508-512.

19. Del Pinal F, Taylor GI. The deep venous system and reverse flow flaps. Br J Plast Surg 1993;46:652-664. 